\title{
Investigation of InSb:Mn thin films magnetoresistance by I-V characteristics method
}

\author{
Oleg Novodvorsky ${ }^{1,}$, , Vladimir Mikhalevsky ${ }^{l}$, Liubov Parshina ${ }^{l}$, Olga Khramova, Andrey Lotin ${ }^{l}$, Elena Cherebylo ${ }^{l}$, \\ Sergey Marenkin ${ }^{2}$, Aleksey Aronov ${ }^{2}$, Aleksey Kochura ${ }^{3}$, and Vladislav Panchenko ${ }^{l}$ \\ ${ }^{1}$ ILIT RAS - Branch of FSRC "Crystallography and Photonics" of RAS, Shatura, Russia \\ ${ }^{2}$ Kurnakov Institute of the general and inorganic chemistry of the RAS, Moscow, Russia \\ ${ }^{3}$ South - West State University, Kursk, Russia
}

\begin{abstract}
We have investigated the effect of magnetoresistance in the thin films of InSb:Mn using the data of I-V curves. The thin films were produced by pulsed laser deposition (PLD) method from the InSb $\mathrm{MnSb}$ composite alloy targets with the concentrations of $\mathrm{MnSb}$ to $20 \%$. The temperature dependence of magnetoresistance of the films has been studied in the temperature range from $10 \mathrm{~K}$ to $300 \mathrm{~K}$.
\end{abstract}

\section{Introduction}

The fabrication of In1-xMnxSb thin films with a uniform distribution of magneto-active component presents a challenge [1]. The investigation of thin films of ferromagnetic semiconductors (AIII-BV):Mn is of great interest in connection with a possibility of their use in the magnetoelectronic and spintronic devices [2]. A bulk sample of the InSb-MnSb composite eutectic represents the narrow band semiconductor of $p$-type that has the lattice parameters approaching those of InSb and is characterized by high mobility of carriers. The Curie temperature in In 1-xMnxSb alloys reaches $600 \mathrm{~K}$. Low solubility of manganese in $\mathrm{InSb}$ is responsible for formation of nanoclusters of $\mathrm{MnSb}$ in bulk sample with concentrations higher than 3,5\% [3]. Though solubility of manganese in InSb is small, the pulsed laser deposition method (PLD) makes possible obtaining homogeneous thin films of InSb:Mn with concentration of manganese to $20 \%$ from the targets of InSb-MnSb alloys. The PLD method allows producing thin films of various materials on single-crystal, amorphous and flexible organic substrates [4]. The presence of drops is the main factor of reduction in the quality of the films obtained by traditional PLD. Application of the dropletfree mode of PLD provides the production of the highquality smooth uniform films on the substrate of room temperature owing to removing of the drops which take off from the target and fall on the substrate in the process of film deposition. In the process of film growth the mechanical separator permits the passage of quickly flying atoms and ions to the substrate, but presents falling of large drops on the growing film. The dropletfree mode of film deposition provides smooth homogeneous high-quality films even at the ambient temperature of the substrate thanks to elimination of the drops which are emitted from the target onto the substrate in the deposition process [5]. The films of the diluted magnetic semiconductors with high Curie temperature and high extent of spin polarization of carriers [6] were obtained by this method. The magnetoresistance in thin films of InSb:Mn can have influence on the characteristics of the magneto-operated $\mathrm{p}$-(InSb+MnSb)/n-InSb diodes in which the effect of giant magnetoresistance is observed [7-9].

\section{Experimental procedure}

The thin InSb:Mn films were grown on sapphire (0001) substrates by ablation with an excimer KrF laser using the droplet-free pulsed laser deposition (PLD) method [4]. The size of substrates was $10 \times 10 \mathrm{~mm}$. The $\mathrm{InSb}+\mathrm{MnSb}$ composite alloy target contained up to $20 \%$ of $\mathrm{MnSb}$. The composition homogeneity and the morphology of the films were investigated by the X-ray spectroscopy, the SEM method with an attachment for energy-dispersive X-ray spectroscopy, by X-ray phase analysis (XPS), and by the AFM method with a magnetic cantilever. We used Hall method to measure the conductivity type of the charge carriers in the films. The films exhibited p-type conductivity. The thickness of the films was determined with the aid of the MII-4 interferometer.

\section{Results and discussion}

The investigation of the films with SEM showed the homogeneous distribution of the In, Sb and Mn elements in the surface layer of the film. According to the results of energy-dispersive X-ray spectroscopy, the concentration of these elements corresponded to the composition of the initial target. We deposited 10 films with concentration of manganese from $3,5 \%$ (eutectic) up to $20 \%$. Thickness of the films was $50-80 \mathrm{~nm}$. The results of the AFM analysis using a magnetic cantilever point to the presence of ferromagnetic clusters of the size of $4 \mathrm{~nm}$ of our thin films of InSb:Mn with concentration

* Corresponding author: onov@mail.ru 
of manganese $20 \%$. The InSb: Mn films with $\mathrm{MnSb}$ concentration of $3,5 \%$ (eutectic) were examined with the help of SEM and AFM, which showed the homogeneity of the distribution of elements on the surface of the InSb:Mn film. X-ray diffraction studies of the InSb:Mn film on a Bruker D8 Advance diffractometer showed the presence of very weak MnSb peak (Fig.1).

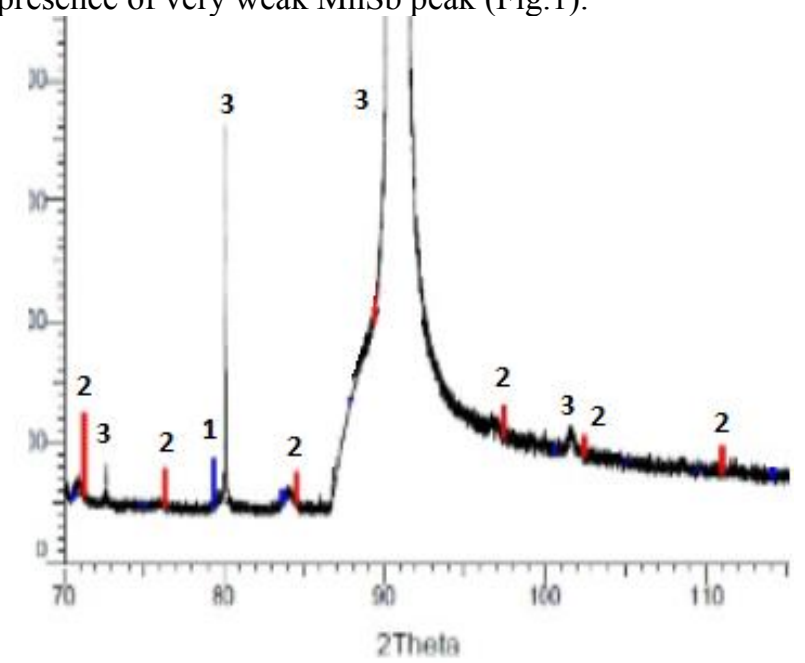

Fig.1. X-ray pattern of an InSb:Mn film with $\mathrm{MnSb}$ concentration of $3,5 \%$ (eutectic) on a sapphire substrate: 1 MnSb phase, 2 - InSb phase, 3 - InMnSb phase (eutectic).

\subsection{Magnetoresistance of thin InSb:Mn films}

In this paper, we have for the first time investigated the magnetoresistive effect in the thin InSb:Mn films using the $I-V$ curve method. Thin films of InSb:Mn ferromagnetic semiconductors are widely investigated $[1,7]$. However, the papers does not show the dependence of the magnetoresistance of films on the voltage applied in the plane of the film. Such dependencies can be obtained from the I-V characteristic. The magnetoresistance of the $p$-(InSbMn) films with $\mathrm{MnSb}$ concentration of $3,5 \%$ (eutectic) was measured under the constant magnetic field of two values, $0.15 \mathrm{~T}$ and $1 \mathrm{~T}$. The measurements were made under room temperature. The field of $0.15 T$ was applied collinearly to the current flowing in the film (Fig.2), and the field of $0.15 \mathrm{~T}$ or the field of $1 \mathrm{~T}$ was applied perpendicular to the film plane and to the current flowing in the film (Fig.3). The indium ohmic contacts were formed on the film surface before measuring of the I-V curve using the Keithley 2612 device. The $I-V$ curve was measured in the range of applied voltage from $-10 \mathrm{~V}$ to $+10 \mathrm{~V}$ in steps of $1 \mathrm{mV}$. Switching of the voltage polarity allowed reversing the polarity of the electric field. Figures 2, 3 illustrates the dependence of the magnetoresistance of the $p$-InSb:Mn thin films on the voltage for collinear and perpendicular electric and magnetic fields under room temperature. The magnetoresistance MR (U) was measured on the basis of $I-V$ curve in the temperature range from $10 \mathrm{~K}$ to $300 \mathrm{~K}$ using the formula $(R(H)-R(0)) / R(0) * 100 \%$, where $R(0)$ and $R(H)$ are the resistances in the absence and presence of a magnetic field, respectively. It was observed that the magnetoresistance of the sample was negative and depended on the magnetic field orientation with respect to the current direction at room temperature.

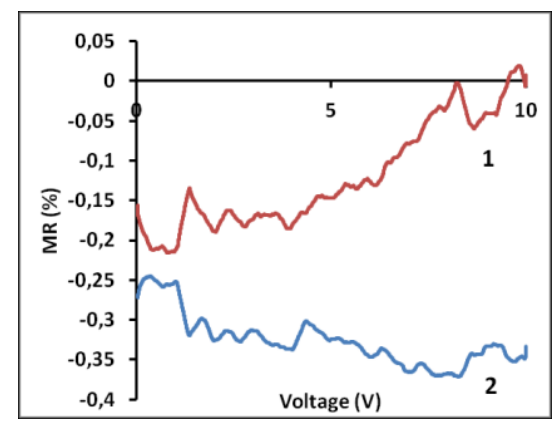

Fig.2. The magnetoresistance of the $p$-InSb:Mn thin films vs the voltage for collinear electric and magnetic fields located in the plane of the film under room temperature $(1-E \uparrow \downarrow B$, $2-E \uparrow \uparrow B), B=0.15 \mathrm{~T}$.

The observed different behavior of the magnetoresistance of the films depending on mutual orientation of the collinear magnetic and electric fields can be caused by different mean free paths of the charge carriers for the parallel and antiparallel directions of the fields $\mathrm{E}$ and $\mathrm{H}$ in the thin InSb:Mn films caused by the presence of surface magnetic nanoclusters, which are registrated by the AFM with a magnetic cantilever.

The measurements were made of the dependence of the film magnetoresistance on the voltage for the magnetic field perpendicular to the film surface for two magnetic field values $B=0.15 \mathrm{~T}$ and $B=1 \mathrm{~T}$. The electric field is localized within the film plane (Fig.3).

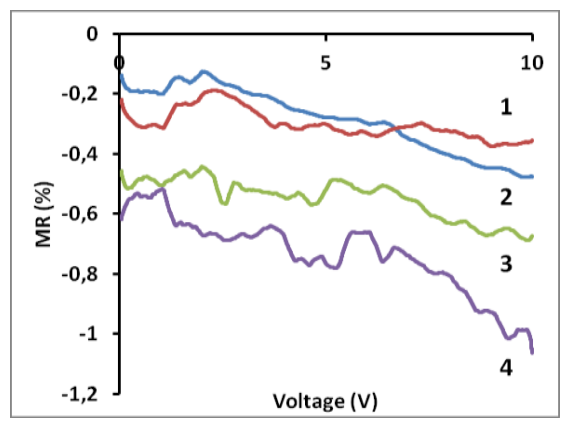

Fig.3. The magnetoresistance of the film vs the voltage for the magnetic field perpendicular to the film under room temperature $(1-B=0,15 \mathrm{~T} \uparrow, 2-B=0,15 \mathrm{~T} \downarrow, 3-B=1 \mathrm{~T} \uparrow$, $4-B=1 \mathrm{~T} \downarrow)$.

For the magnetic field normal to the film a similar dependence on the magnitude of the field is disclosed [7].

The temperature dependence of the magnetoresistance of p-InSb:Mn films with the MnSb concentration of $20 \%$ was studied in the temperature range from $10 \mathrm{~K}$ to 300 $\mathrm{K}$. The investigations were carried out by measuring and analyzing their I-V curves in the temperature range from $10 \mathrm{~K}$ to $300 \mathrm{~K}$. The measurement wires were connected to the indium ohmic contacts which were formed on the surface of each film. The films were placed in a cryostat. The measurements of the I-V curves were performed in the field with permanent magnet and without it. The dependences of the magnetoresistance for a fixed temperature and magnetic field were calculated on the 
basis of the $\mathrm{I}-\mathrm{V}$ curve. The results of measurements $\mathrm{R}$ and calculations MR are shown in Fig. 4, 5.

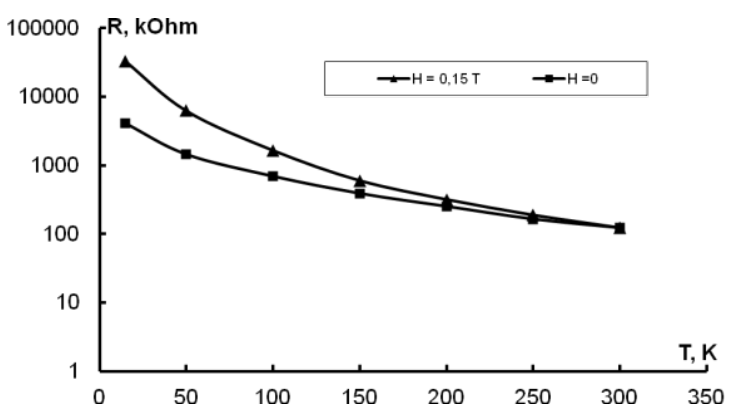

Fig.4. The dependence of the film InSb:Mn resistance on the temperature in the absence (squares) and in the presence of a magnetic field of $0.15 \mathrm{~T}$ (triangles). The voltage applied to the sample is $10 \mathrm{~V}$.

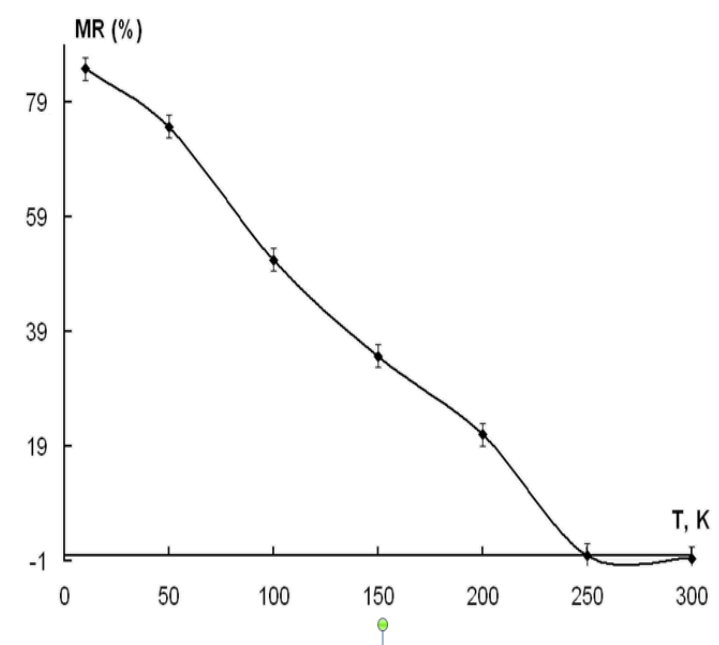

Fig. 5. The dependence of the thin InSb:Mn film magnetoresistance on the temperature at an applied voltage of $10 \mathrm{~V}$ and under magnetic field of $0.15 \mathrm{~T}$ in the plane of the film.

Figure 5 shows that the crossover from positive to negative giant magnetoresistance (GMR) is observed at the temperature of $250 \mathrm{~K}$ during cooling. As the sample cooled down, the value of GMR increased and reached $80 \%$ at $15 \mathrm{~K}$ in the magnetic field of $0.15 \mathrm{~T}$ directed perpendicular to the plane of the film. Thus, it has been shown that the magnetoresistance of the InSb:Mn thin films is registered even at room temperature under small magnetic fields, which opens up considerable prospects for using this material to create various spintronic devices.

\section{Conclusion}

The temperature dependence of the magnetoresistance of InSb: Mn films is studied. In the temperature range of the film from room temperature to $250 \mathrm{~K}$, a negative magnetoresistance was observed. At $250 \mathrm{~K}$, a transition from a negative giant magnetoresistance (GMR) to a positive one was observed. As the sample cooled down, the GMR increased and reached $80 \%$ at $15 \mathrm{~K}$ in a magnetic field of $0.15 \mathrm{~T}$ directed perpendicular to the plane of the film. The magnetoresistance of InSb: Mn thin films is recorded even at room temperature in small magnetic fields, which opens the prospect of using this material to create various spintronics devices.

The work was supported by the Federal Agency of Scientific Organizations (Agreement No 007-ГЗ/Ч3363/26) in part of films deposition and by the Russian Foundation for Basic Research (projects No. 15-07-03580,15-29-01171, 16-2905385, 16-07-00657, 16-07-00842) in part films analysis, pr. of ONIT RAS (code IV.3.3) and Presidium RAS (Code I.III) in part of numerical simulation.

\section{References}

1. N. D. Parashar, N. Rangaraju, V. K. Lazarov, S. Xie, B. W. Wessels, Phys. Rev. B 81, 115321 (2010)

2. A.V. Kochura, B. A. Aronzon, K. G. Lisunov, A. V. Lashkul, A. A. Sidorenko, R. De Renzi, S. F. Marenkin, M. Alam, A. P. Kuzmenko, and E. Lahderanta, JAP 113, 083905 (2013)

3. S.F. Marenkin, A.V. Kochura, I.V. Fedorchenko, A.D. Izotov, M.G.Vasil'ev, V.M.Trukhan, T.V.Shelkovaya, O.A. Novodvorsky, A.L. Zheludkevich, Inorganic materials 52, № 3, 268 (2016)

4. O.A. Novodvorskiy, V.Ya. Panchenko, in Collective monograph "Modern laser-information technologies", ed. Acad. V.Ya. Panchenko and prof. F.V. Lebedev - Moscow: Publishing house Intercontact Science, - 959 p. (2015)

5. L.S. Parshina, O.A. Novodvorsky, O.D. Khramova, I.A. Petukhov, V.A. Mikhalevsky, A.A. Lotin, E.A. Cherebilo, V.Ya. Panchenko, Optical and Quantum Electronics 48, 316(9) (2016)

6. V.V. Rylkov, A.S. Bugaev, O.A. Novodvorskii, V.V. Tugushev, E.T.Kulatov, A.V. Zenkevich, A.S. Semisalova, S.N. Nikolaev, A.S. Vedeneev, A.V. Shorokhova, D.V. Aver'yanov, K.Yu. Chernoglazov, E.A. Gan'shina, A.B. Granovsky, Y. Wang, V.Ya. Panchenko, S. Zhou, JMMM 383, 39 (2015)

7. J.A. Peters, N. Rangaraju, C. Feeser and B. W. Wessels, Appl. Phys. Lett, 98, 193506 (2010)

8. O.D. Khramova, V.A. Mikhalevsky, L.S. Parshina, O.A. Novodvorsky, S.F.Marenkin, A.A. Lotin, E.A. Cherebilo, B.A. Aronzon, A.N. Aronov, V.Ya. Panchenko, Optical and Quantum Electronics. 48, 361(8) (2016)

9. Peters et al. PHYSICAL REVIEW B 82, 205207 (2010) 